\title{
Calculating eGFR Using Apparent Diffusion Coefficient (ADC) Values Obtained Through MR Imaging
} Jaturat Kanpittaya, ${ }_{1}^{1}$ Patrawan Jaimook, ${ }^{1}$ Theerayut Thongkrau, ${ }^{2}$ Chaiyapon Keeratikasikorn, ${ }^{2}$ and Kittisak Sawanyawisuth ${ }^{3,4,5, *}$

${ }^{1}$ Department of Radiology, Faculty of Medicine, Khon Kaen University, Khon Kaen, Thailand

${ }^{2}$ Department of Computer Sciences, Faculty of Sciences, Khon Kaen University, Khon Kaen, Thailand

${ }^{3}$ Department of Medicine and Ambulatory, Medicine Research Group, Faculty of Medicine, Khon Kaen University, Khon Kaen, Thailand

${ }^{4}$ Research Center in Back, Neck Other Joint Pain and Human Performance (BNOJPH), Khon Kaen University, Khon Kaen, Thailand

${ }^{5}$ Sleep Apnea Research Group, Research and Training Center for Enhancing Quality of Life of Working-Age People, Khon Kaen University, Khon Kaen, Thailand

"Corresponding author: Kittisak Sawanyawisuth, MD, PhD, Department of Medicine, Faculty of Medicine, Khon Kaen University, Khon Kaen, 40002, Thailand. Tel: +66-43363664, Fax: +66-43348399, E-mail: kittisak@kku.ac.th

Received 2017 May 18; Accepted 2017 October 09.

\begin{abstract}
Background: Renal dysfunction is associated with significantly lower apparent diffusion coefficient (ADC) values. There are several ADC-level cutoff points that indicate renal dysfunction.

Objectives: This study aimed to evaluate whether or not ADC values can predict estimated glomerular filtration rate (eGFR) levels. If so, the formula to calculate eGFR level by ADC levels will be formulated.

Patients and Methods: We reviewed all adult patients who underwent upper abdomen MRI and magnetic resonance cholangiopancreatography (MRCP) protocol from January 2014 to October 2014. The ADC values and eGFR levels were recorded. The multivariate linear regression was implemented between the eGFR and studied variables. The formula to predict eGFR was produced by the final model of multivariate linear regression analysis. An online formula for eGFR prediction was also created.

Results: There were 261 patients who met the study criteria of which 133 patients were male (51.10). The mean age and body weight (S.D.) of all patients was 59.89 years (13.73) and $55.92 \mathrm{~kg}$ (11.17), respectively. The majority of patients had an eGFR of $60-89 \mathrm{~mL} / \mathrm{min}$ (107 patients; $41.00 \%$ ). The average ADC value of all patients was $1.87 \times 10^{-3} \mathrm{~mm}^{2} / \mathrm{s}$. There were four factors that were significantly associated with eGFR by multivariate linear regression analysis: age, sex, body weight, and ADC value. ADC value had the highest estimates at 121.94 .

Conclusion: ADC values can predict eGFR using the following formula: estimated eGFR $=-139.03-(0.80 \times$ age $)-(4.19 \times$ sex; [0 if female and 1 if male $])+0.57$ (body weight in $\mathrm{kg})+(121.94 \times$ ADC). This formula was also created as an online tool for both mobile and computer at http:/|202.28.94.20/gfr/.
\end{abstract}

Keywords: Diffusion Weight Image, Apparent Diffusion Coefficient, Renal Function, Formula, Online

\section{Background}

Chronic kidney disease (CKD) is a major public health problem. Earlier stages of CKD can be detected through routine laboratory assessments including measurement of serum creatinine and urinalysis (1). The estimated glomerular filtration rate (eGFR) can be calculated by serum creatinine, weight, and ethnicity (1). Radiographic studies are additional helpful tools in identifying renal parenchymal diseases, particularly pertaining to the function of each kidney (2-4).

Diffusion weighted (DW) MRI is a technique to evaluate the diffusion process of water molecules, which is dependent on the cellular environment. Quantitative values of diffusion can be measured from diffusion coefficient im- ages and are called apparent diffusion coefficient (ADC) values. These combine the effect of capillary perfusion and water diffusion. DW MRI in kidneys is suitable due to the high blood flow and water filtration. The DW MRI in renal diseases is an evolving field and previous investigators have attempted to evaluate its utility in the characterization of focal renal lesions, renal parenchymal disease, and renal infections (5).

Renal dysfunction is associated with significantly lower ADC values in the cortex and medulla than in those of healthy subjects $(6,7)$. An ADC cutoff point at lower than $2.035\left(\times 10^{-3} \mathrm{~mm}^{2} / \mathrm{s}\right)$ indicated renal dysfunction, while an ADC over $2.452\left(\times 10^{-3} \mathrm{~mm}^{2} / \mathrm{s}\right)$ is found in cases of normal renal function. There are several ADC-level cutoff points that indicate renal dysfunction $(8,9)$. 


\section{Objectives}

Other than the role of indicating renal function of the ADC level, this study aimed to evaluate whether or not ADC values can predict eGFR levels. If so, the predictive formula will be created to show the association of ADC and eGFR.

\section{Patients and Methods}

\subsection{Study Population}

This was a retrospective study. We reviewed all adult patients who underwent upper abdomen MRI and magnetic resonance cholangiopancreatography (MRCP) protocol from January 2014 to October 2014.

Patients whose kidneys were unsuitable for ADC measurement, such as those with multiple cystic lesions or that were very small, were excluded. Clinical factors including eGFR were traced back in all eligible patients.

The study protocol was approved by the institutional review board of ethical issues, Khon Kaen University.

\subsection{Imaging Techniques}

Two hundred and sixty patients were imaged using a 3T MR scanner (Phillips Achieva; Philips, Best, the Netherlands) and a 1.5T MR scanner (MAGNETOM Aera; Siemens, Erlangen, Germany). Imaging was performed using a phased array body coil with the patient in supine position. The abdominal imaging protocol included True Fast Imaging and Steady Precession (True FISP) axial and coronal sequences, which served as localizer for planning further sequences. Following this, conventional MRI sequences, T1W axial (in and opposed phase) sequences and fat-suppressed (FS) T2W axial and coronal sequences were acquired. The following parameters were used for the echo planar image (EPI): two dimension diffusion weighted imaging (DWI) in three $b$ values $(0,150,800)$ trigger fat suppression (FS) or spectral attenuated inversion recovery (SPAIR) thickness 6 $\mathrm{mm}, 32$ - 36 slices, field of view (FOV) $340 \times 100 \mathrm{~mm}$ and $340 \times 274 \mathrm{~mm}$ on $1.5 \mathrm{~T}$ and $3 \mathrm{~T}$, respectively. Scan time was 3.34 minutes: matrix $124 \times 137$ repetition time (TR)/echo time $(\mathrm{TE})=1700 / 71 \mathrm{~ms}$ on axial DW images of 1.5T MR and 2.37 minutes, matrix $124 \times 137 \mathrm{TR} / \mathrm{TE}=250 / 53 \mathrm{~ms}$ with flip angle 90 degrees on 3T MR were performed. The ADC maps were automatically generated from the datasets of DW images using the operating console and ADC values were calculated.

\subsection{Image Analysis}

A workstation used in this study was produced by the Fujifilm Medical Systems, USA, Inc. Radiological analysis was performed by the same radiologist in all patients.
A circular $1.0 \mathrm{~cm}^{2}$ region of interest (ROI) at the corticomedullary junction was used to collect the ADC values (Figure 1). For each kidney, one ROI was placed in the middle portion of the kidneys, which is less influenced by the perfusion effect. The measurement was performed to avoid the edges of the border of the kidney to exclude the effect of partial volume averaging.

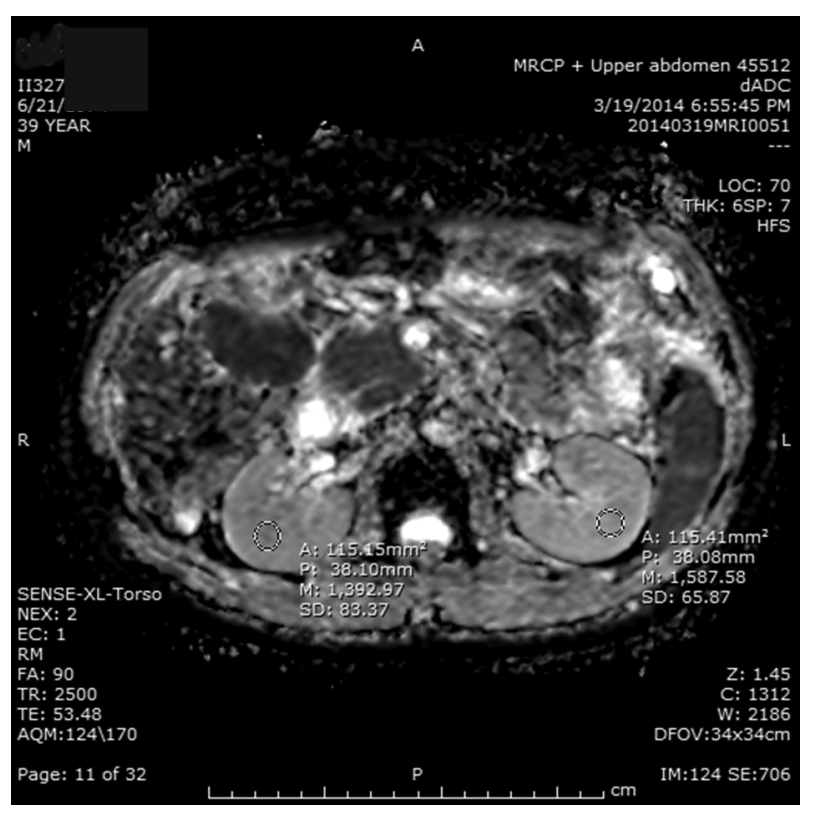

Figure 1. The diffusion-weighted imaging and the apparent diffusion coefficient (ADC) values in a 39-year-old man with hepatocellular carcinoma (HCC) at segment $\mathrm{VI} / \mathrm{VII}$ showed the ADC values of $1.392\left(\times 10^{-3} \mathrm{~mm}^{2} / \mathrm{s}\right)$ in the right kidney and $1.587(\mathrm{x}$ $\left.10^{-3} \mathrm{~mm}^{2} / \mathrm{s}\right)$ in the left kidney. The mean of ADC value is $1.489\left(\times 10^{-3} \mathrm{~mm}^{2} / \mathrm{s}\right)$.

\subsection{Statistical Analysis}

Descriptive statistics were used to calculate all studied variables including age, sex, body weight, serum creatinine, eGFR, and the ADC values. The scatter plot of eGFR and the ADC was plotted, as was Pearson correlation between both factors. The univariate and multivariate linear regression were computed between the eGFR and studied variables. The formula to predict the eGFR was produced by the final model of multivariate linear regression analysis. An online formula for eGFR prediction was also created. All statistical analyses were performed using SAS software.

\section{Results}

There were 261 patients who met the study criteria, of which 133 patients were male (50.96\%). The mean age and body weight (S.D.) of all patients was 59.89 years (13.73) and $55.92 \mathrm{~kg}$ (11.17), respectively. The majority of patients had 
eGFR at 60 - $89 \mathrm{~mL} / \mathrm{min}$ (107 patients; 41.00\%). The average ADC value of all patients was $1.87 \times 10^{-3} \mathrm{~mm}^{2} / \mathrm{s}$, as shown in Table 1.

\begin{tabular}{|c|c|}
\hline Factors & Values \\
\hline Age, year & $59.89 \pm 13.73$ \\
\hline Male, sex & $133(50.96)$ \\
\hline Body weight, kg & $55.92 \pm 11.17$ \\
\hline Serum creatinine, $\mathrm{mg} / \mathrm{dL}$ & $1.01 \pm 0.97$ \\
\hline eGFR, $\mathrm{mL} / \mathrm{min}$ & $71.05 \pm 32.37$ \\
\hline$>90$ & $56(21.46)$ \\
\hline $60-89$ & $107(41.00)$ \\
\hline $30-59$ & $78(29.89)$ \\
\hline $15-29$ & $15(5.75)$ \\
\hline$<15$ & $5(1.92)$ \\
\hline \multicolumn{2}{|l|}{$\mathrm{ADC}, \times 10^{-3} \mathrm{~mm}^{2} / \mathrm{s}$} \\
\hline Right kidney & $1.86 \pm 0.17$ \\
\hline Left kidney & $1.89 \pm 0.17$ \\
\hline Mean & $1.87 \pm 0.15$ \\
\hline
\end{tabular}

Abbreviation: eGFR, estimated glomerular filtration rate.

${ }^{a}$ Values are expressed as mean \pm SD or No. (\%)

The Pearson correlation coefficient between the ADC value and eGFR was 0.7882 (P value $<0.001$ ). Figure 2 shows the scatter plot of the correlation of both factors. There were four factors that were included in the univariate and multivariate linear regression analysis to estimate eGFR: age, sex, bodyweight, and ADC value. Three of these factors (all except sex) were shown to be significant by univariate linear regression analysis. However, all factors were shown to be significantly associated with eGFR by multivariate linear regression analysis. ADC value had the highest estimates at 121.94 (Table 2). The adjusted R square of the multivariate model was 0.7395 .

\section{Discussion}

This study showed another role of ADC levels to predict eGFR, other than just the cutoff point for renal dysfunction. The ADC values can predict eGFR by using the formula from a multivariate linear regression model. The predicted eGFR $=-139.03-(0.80 \times$ age $)-(4.19 \times$ sex; 0 if female and 1 if male $)$ +0.57 (body weight in $\mathrm{kg})+(121.94 \times$ ADC). This formula was also created as an online tool at http:/|202.28.94.20/gfr/ and the eGFR can be calculated by filling in these four variables.

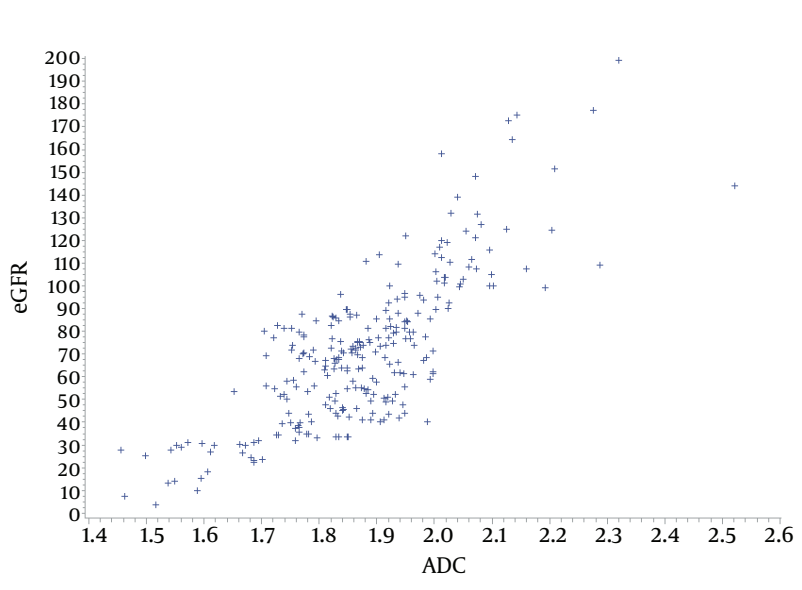

Figure 2. The scatter plot of estimated glomerular filtration rate (eGFR) in $\mathrm{mL} / \mathrm{min}$ and the apparent diffusion coefficient (ADC) in $\times 10^{-3} \mathrm{~mm}^{2} / \mathrm{s}$.

The ADC values were also correlated with the eGFR linearly (Figure 2) as previously reported (9). The Pearson correlation index in this study was slightly higher than a similar study from China (0.7882 vs 0.709). The high association of the ADCvalues and eGFR was positively correlated with the estimate of 121 by multivariatelinear regression analysis (Table 2). As mentioned earlier, the ADC measurements are directly associated with the Brownian motion of water molecules, capillary perfusion, and tubular flow.

Age is one factor that affects renal function. After the age of 40 , the eGFR starts to decline by approximately 8 $\mathrm{mL} / \mathrm{min}$ per decade. Our formula confirmed this theory (10). With an increase in age of one year, eGFR decreases by $0.80 \mathrm{~mL} / \mathrm{min}$ or exactly $8 \mathrm{~mL} / \mathrm{mim}$ per decade (Table 2). Male sex was another factor independently and negatively associated with eGFR, even though it was not a significant factor according to univariate linear analysis. Males tended to have a four times lower eGFR compared with female patients. Additionally, men seemed to have more symptoms of CKD than women. In male patients with eGFR $20-30 \mathrm{~mL} / \mathrm{min}$, the hematocrit was lowered by $9.4 \%$, while it was only lowered by $5.3 \%$ in female patients with the same eGFR level (11).

For every $1 \mathrm{~kg}$ increase in bodyweight eGFR was increased by $0.57 \mathrm{~mL} / \mathrm{min}$ (Table 2). Previous studies showed that obesity increased renal blood flow and also eGFR (12, 13). The hyperfiltration in obese patients may result in renal dysfunction later on. In this study, the correlation between body weight and eGFR may be explained by the fact that those patients with renal dysfunction had lower body weight than those with higher eGFR, as has previously been reported (14). Body weight in this study may not indicate a body mass index. 
Table 2. Prediction of Estimated Glomerular Filtration Rate by a Number of Variables Using Univariate and Multivariate Linear Regression Analysis

\begin{tabular}{lccc}
\hline Variables & Estimates $^{\mathbf{a}}$ & PValue $^{\mathbf{a}}$ & Estimates $^{\mathbf{b}}$ \\
\hline Intercept & $\mathrm{NA}$ & $\mathrm{NA}$ & -139.03 \\
Age & -1.60 & $<0.001$ & -0.80 \\
Sex & -0.57 & 0.887 & -4.19 \\
Body weight & 1.04 & $<0.001$ & 0.001 \\
ADC & 170.94 & $<0.001$ & 0.57 \\
\hline
\end{tabular}

Abbreviations: ADC, apparent diffusion coefficient; NA, not applicable.

${ }^{\mathrm{a}}$ Univariate linear regression analysis.

${ }^{\mathrm{b}}$ Multivariate linear regression analysis.

Radiologists are now able to estimate the patients'eGFR during MRI procedures even without knowing the patients' serum creatinine levels (14). Moreover, the radiologists may be able to calculate the eGFR of each kidney separately (Table 1). Another advantage is the ability to use an online tool for eGFR calculation based on ADC values. Therefore, radiologists can calculate the eGFR of each kidney and indicate renal dysfunction in patients who routinely performed abdominal MRI for non-renal indications without knowing serum creatinine. Renal pathology was also evaluated in addition to the eGFR.

There are some limitations to this study. The formula may not be universal for all eGFR levels. Most patients in this study were patients with eGFR above $30 \mathrm{~mL} / \mathrm{min}$ (92.34\%). Only 7.67\% of patients had eGFR less than 30 $\mathrm{mL} / \mathrm{min}$ (Table 1 ). The formula may not be suitable for other ethnicities. Further studies should be performed in other particular ethnicities to formulate the formula.

In conclusion, $\mathrm{ADC}$ values can predict eGFR using the following formula: estimated eGFR $=-139.03-(0.80 \times$ age $)$ - $(4.19 \times$ sex; [0 if female and 1 if male] $)+0.57$ (body weight in $\mathrm{kg})+(121.94 \times \mathrm{ADC})$. This formula was also created as an online tool for both mobile and computer at http://202.28.94.20/gfr/.

\section{Acknowledgments}

The authors would like to thank Mr. Dylan Southard for his English language editing and the Thailand research fund (TRF) for their kind support (IRG 5780016). This research was also funded in part from grant of Faculty of Medicine, Khon Kaen University, Thailand (Grant Number RG593011); the higher education research promotion national research University project of Thailand, office of the higher education commission through the health cluster (SHeP-GMS), Thailand; the faculty of medicine, Khon Kaen University grant number TR57201; and the TRF senior re- search scholar grant, Thailand research fund grant number RTA5880001.

\section{Footnote}

Conflict of Interests: None declared by all authors.

\section{References}

1. National Kidney F. K/DOQI clinical practice guidelines for chronic kidney disease: evaluation, classification, and stratification. Am J Kidney Dis. 2002;39(2):1-266. [PubMed: 11904577].

2. Bauer JH, Brooks CS, Burch RN. Clinical appraisal of creatinine clearance as a measurement of glomerular filtration rate. Am J Kidney Dis. 1982;2(3):337-46. [PubMed: 7148824].

3. Prigent A. Monitoring renal function and limitations of renal function tests. Semin Nucl Med. 2008;38(1):32-46. doi: 10.1053/j.semnuclmed.2007.09.003. [PubMed:18096462].

4. Chandarana H, Lee VS. Renal functional MRI: Are we ready for clinical application?. AJR Am J Roentgenol. 2009;192(6):1550-7. doi: 10.2214/AJR.09.2390. [PubMed:19457818].

5. Squillaci E, Manenti G, Di Stefano F, Miano R, Strigari L, Simonetti G. Diffusion-weighted MR imaging in the evaluation of renal tumours.J Exp Clin Cancer Res. 2004;23(1):39-45. [PubMed: 15149149].

6. Goyal A, Sharma R, Bhalla AS, Gamanagatti S, Seth A. Diffusionweighted MRI in assessment of renal dysfunction. Indian J Radiol Imaging. 2012;22(3):155-9. doi: 10.4103/0971-3026.107169. [PubMed: 23599561].

7. Carbone SF, Gaggioli E, Ricci V, Mazzei F, Mazzei MA, Volterrani L. Diffusion-weighted magnetic resonance imaging in the evaluation of renal function: a preliminary study. Radiol Med. 2007;112(8):1201-10. doi: 10.1007/s11547-007-0217-6. [PubMed:18074195].

8. Toya R, Naganawa S, Kawai H, Ikeda M. Correlation between estimated glomerular filtration rate (eGFR) and apparent diffusion coefficient (ADC) values of the kidneys. Magn Reson Med Sci. 2010;9(2):59-64. [PubMed: 20585195].

9. Xu Y, Wang X, Jiang X. Relationship between the renal apparent diffusion coefficient and glomerular filtration rate: preliminary experience. J Magn Reson Imaging. 2007;26(3):678-81. doi: 10.1002/jmri.20979. [PubMed: 17729335].

10. Rowe JW, Andres R, Tobin JD, Norris AH, Shock NW. The effect of age on creatinine clearance in men: a cross-sectional and longitudinal study. J Gerontol. 1976;31(2):155-63. [PubMed: 1249404]. 
11. Hsu CY, Bates DW, Kuperman GJ, Curhan GC. Relationship between hematocrit and renal function in men and women. Kidney Int. 2001;59(2):725-31. doi: 10.1046/j.1523-1755.2001.059002725.x. [PubMed: 11168955].

12. Chagnac A, Weinstein T, Herman M, Hirsh J, Gafter U, Ori Y. The effects of weight loss on renal function in patients with severe obesity. JAm
Soc Nephrol. 2003;14(6):1480-6. [PubMed: 12761248].

13. Bolignano D, Zoccali C. Effects of weight loss on renal function in obese CKD patients, a systematic review. Nephrol Dial Transplant. 2013;28 Suppl 4:82-98. doi:10.1093/ndt/gft302. [PubMed: 24092846].

14. Cockcroft DW, Gault MH. Prediction of creatinine clearance from serum creatinine. Nephron. 1976;16(1):31-41. [PubMed: 1244564]. 